\title{
Impatto del centro diurno sui costi di assistenza di pazienti affetti da alzheimer probabile, un'esperienza della asl di Città di Castello
}

Trequattrini*A., Guidi** L., Cestini*** R., Mezzetti*M., Ciappi*F.

\begin{abstract}
Objective of the present work is to evaluate the economic impact caused by the attendance of the Local Health Care Unit Diurnal Center (DC) by Alzheimer disease patients. 21 Alzheimer patients were observed prospectively during 8 months: 2 months before DC attendance (period 1), 4 months during DC attendance (period 2) and finally 2 months after DC discharge (period 3). Data collected were analyzed and average resources consumption was translated into monetary costs according to the Local Health Care Unit point of view and family burden.

Our analysis showed a significant cost reduction during DC attendance in respect to period 1 and 3 , both on Local Health Care Unit and family sides. During period 2 (DC attendance) patients showed a significant clinical improvement too, as demonstrated by MMSE (Mini Mental State Examination), ADL (Activity of Daily Leaving), IADL (Instrumental Activity of Daily Leaving) scales.

Finally our work demonstrates that the DC is a valid and economically advantageous support for the health care assistance of Alzheimer patients on SSN side and that it is able to reduce the burden of continuous care giving for families.
\end{abstract}

Farmeconomia e percorsi terapeutici 2004; 5 (1): $25-29$

\section{INTRODUZIONE}

I dati demografici inquadrano l'Italia come uno dei paesi a più rapido invecchiamento. Nel 1995 gli ultrasessantacinquenni rappresentavano il $16 \%$ della popolazione generale Italiana, circa 9 milioni di individui, contro il $6 \%$ riconosciuto come media a livello mondiale. Le stime ISTAT di evoluzione e crescita della popolazione italiana vedono un incremento della popolazione anziana, nel prossimo decennio, significativamente superiore a quella internazionale con un $20,6 \%$ di ultrasessantacinquenni nel 2010 e un tasso di crescita annuo del 16,3 per mille abitanti [1].

Attualmente si stima che circa 500.000 persone anziane siano affette da demenza in Italia, per un costo complessivo di assistenza (a carico di SSN e famiglie) di circa 29.737 miliardi di lire (15,36 miliardi di euro) nel 1995 (1,47\% del PIL) [2]. Il costo individuale medio della demenza nel nostro paese, stabilito dagli stessi autori, è di circa 50.612,77 euro/anno.
Una delle esperienze più recenti di valutazione cost of illness della demenza svolta nel nostro paese è rappresentata dallo studio Co.Dem. (Costi della Demenza) [6].

Questo studio longitudinale, condotto tra il 1994 e il 1999, ha analizzato il livello assistenziale resosi necessario per la gestione sanitaria di 110 pazienti affetti da demenza. Il campione di studio è stato stratificato secondo una serie di predittori clinici (livello di gravità della malattia, stato cognitivo e livello di autonomia funzionale), e ha analizzato il carico sia dell'assistenza formale sia di quella informale. Quest'ultima, quasi esclusivamente a carico della famiglia, è risultata rappresentare oltre il 50\% dei costi totali. Il rapporto tra costi per la famiglia e costi per il Sistema Sanitario non cambia significativamente anche quando il paziente, a causa della gravità della malattia e della perdita di autonomia funzionale, viene istituzionalizzato. Le conclusioni dello studio Co.Dem. permettono di individuare nell' autonomia funzionale un importante predittore dei costi, da
* Dipartimento Tutela della Salute Mentale, ASL1 Umbria, Città di Castello

$(P G)$

** Health Outcomes Research, Eli Lilly Italia S.p.A., Sesto Fiorentino (FI) *** Centro Salute, ASL 1 Umbria, Città di Castello (PG) 
mettersi, come lo stato cognitivo, in relazione all'incremento di sopravvivenza.

Nel nostro studio invece ci siamo concentrati particolarmente nel valutare quanto il ricorso a strutture sanitarie di supporto come il Centro Diurno consenta una ottimizzazione degli aspetti di gestione sanitaria e famigliare. Per i dati di utilizzo di risorse sanitarie e di assorbimento di quelle famigliari, ci siamo riferiti ad un recente studio osservazionale condotto su 21 pazienti affetti da demenza tipo Alzheimer probabile presso la ASL 1 di Città di Castello [5].

\section{MATERIALIEMETODI}

Lo studio osservazionale cui abbiamo fatto riferimento per la nostra analisi [5] ha raccolto prospetticamente dati relativi al consumo di risorse sanitarie per il trattamento di 21 pazienti affetti da demenza tipo Alzheimer probabile (secondo i criteri NINCDS-ADRDA), durante un periodo di 8 mesi. Di questi, 4 mesi erano di osservazione del trattamento sul territorio ( 2 mesi prima e 2 mesi dopo l'accesso al Centro Diurno), mentre 4 mesi erano di osservazione durante la frequentazione del Centro Diurno.

Scopo della ricerca era definire il carico assistenziale in termini di numero di prestazioni sanitarie e di impegno della famiglia nelle fasi di assistenza sul territorio e durante la frequentazione del Centro Diurno della ASLe valutare l'impatto che questa struttura può avere sia sull'intensità di assistenza sia sulla progressione della malattia.

L'evoluzione clinica dei pazienti era stata monitorata con le visite programmate nel protocollo, durante le quali venivano somministrati i seguenti test clinici: MMSE (Mini Mental State Examination), ADL (Activity of Daily Leaving), IADL (Instrumental Activity of Daily Leaving).

I dati di assistenza sanitaria raccolti nello studio erano costituiti da: visite mediche specialistiche in ambulatorio e domiciliari, interventi infermieristici in ambulatorio e domiciliari, riabilitazione, psicoterapia, assistenza sociale, ricoveri ospedalieri e accesso al Centro Diurno e carico familiare (espresso come giornate e ore dedicate al paziente assistito, in relazione alla posizione lavorativa del caregiver).

Nella attuale analisi abbiamo convertito le prestazioni sanitarie sopraelencate in corrispettivi economici, assumendo il punto di vista della ASL e, cioè, attribuendo i reali costi di erogazione delle prestazioni sanitarie. Per i costi relativi alle prestazioni sanitarie del territorio e ospedaliere ci siamo riferiti alla letteratura che riporta i costi per il setting psichiatrico e neurogeriatrico $[3,4]$, mentre per la valutazione del costo di gestione del Centro Diurno ci siamo rifatti a dati di spesa della ASL 1 di Città di Castello (Tabella 1).

\begin{tabular}{lcccc}
\hline & Ore/die & $\begin{array}{c}\text { Costo orario } \\
\text { (Euro) }\end{array}$ & $\begin{array}{c}\text { Costo unitario } \\
\text { (Euro) }\end{array}$ & $\begin{array}{c}\text { Costo annuo } \\
\text { (Euro) }\end{array}$ \\
\hline Operatori assistenza & 16 & 15,04 & & $57.753,60$ \\
Educatori & 1,5 & 16,97 & $6.110,22$ \\
Animatori & 1,5 & 16,97 & $6.110,22$ \\
Pasti & & & $13.386,56$ \\
Lavanderia pulizie & & & $3.098,74$ \\
Utenze & & & $3.098,74$ \\
Assicurazioni & & & 604,25 \\
Supporto amministrativo & & & $3.098,74$ \\
e sociale & & $-10,33$ & $-19.833,60$ \\
$\begin{array}{l}\text { Retta pagata dai pazienti } \\
\text { (8 pazienti ogni giorno) }\end{array}$ & & & \\
Totale costo annuo & & & $73.427,49$ \\
Centro Diurno & & & \\
$\begin{array}{l}\text { Costo/die per paziente } \\
\text { (Centro Diurno) }\end{array}$ & & & 25,15 \\
\hline
\end{tabular}

Tabella 1

Costi di gestione del Centro Diurno presso la ASL di Città di Castello
Giorni lavorativi pari a 240/anno, per ognuno dei quali 8 pazienti ospitati dal Centro Diurno 


\begin{tabular}{|c|c|c|c|c|c|c|c|}
\hline & \multicolumn{3}{|c|}{$\begin{array}{c}\text { N. medio } \\
\text { interventi/paziente }\end{array}$} & \multirow{2}{*}{$\begin{array}{c}\text { Costo } \\
\text { unitario/intervento } \\
\text { (Euro) } \\
2003\end{array}$} & \multicolumn{3}{|c|}{$\begin{array}{c}\text { Costi in euro } \\
\text { (media per paziente) }\end{array}$} \\
\hline & T1 & $\mathbf{T 2}$ & T3 & & T1 & $\mathbf{T} 2$ & T3 \\
\hline $\begin{array}{l}\text { Durata periodo } \\
\text { osservazione (mesi) }\end{array}$ & 2 & 4 & 2 & & 2 & 4 & 2 \\
\hline $\begin{array}{l}\text { Visite mediche } \\
\text { ambulatoriali }\end{array}$ & 1,86 & 1,05 & 2,43 & 68,86 & 127,88 & 72,30 & 167,22 \\
\hline Visite mediche domiciliari & 1,29 & 0,00 & 1,20 & 175,63 & 225,81 & 0,00 & 210,76 \\
\hline $\begin{array}{l}\text { Interventi infermieristici } \\
\text { domiciliari }\end{array}$ & 2,00 & 0,83 & 2,05 & 51,09 & 102,18 & 42,58 & 104,61 \\
\hline Supporto assistenza sociale & 1,76 & 1,00 & 2,00 & 82,16 & 144,99 & 82,16 & 164,32 \\
\hline Riabilitazione & 7,33 & 4,00 & 7,25 & 164,04 & $1.202,94$ & 656,15 & $1.189,27$ \\
\hline Ricoveri correlati (giorni)* & 0,00 & 0,00 & 5,00 & $1.550,95$ & 0,00 & 0,00 & $7.754,75$ \\
\hline $\begin{array}{l}\text { Frequentazione } \\
\text { Centro Diurno (giorni)** }\end{array}$ & 0,00 & 60,00 & 0,00 & 25,15 & 0,00 & $1.508,78$ & 0,00 \\
\hline \multicolumn{5}{|l|}{ Totale } & $1.803,80$ & $2.361,97$ & $9.590,94$ \\
\hline \multicolumn{5}{|l|}{ Totale/mese osservazione } & 901,90 & 590,49 & $4.795,47$ \\
\hline \multicolumn{5}{|c|}{ Totale/mese osservazione escludendo il ricovero dopo dimissione dal CD } & 901,90 & 590,49 & 918,09 \\
\hline
\end{tabular}

\section{Tabella 2}

Prestazioni sanitarie e relativi costi di erogazione

Nella Tabella 1 non compaiono i costi di affitto dei locali poiché questi sono stati concessi in comodato gratuito da una locale istituzione. L'occupazione della struttura prevede l'accesso quotidiano, per circa 240 giornate all'anno, di 8 pazienti. Ogni paziente può frequentare il Centro Diurno a giorni alterni dietro la corresponsione di una retta quotidiana di 10,33 euro.

Oltre ai costi sanitari abbiamo valutato anche i costi di assistenza che ricadono sulle famiglie di questi pazienti. In particolare, abbiamo considerato come costi il numero medio di giornate lavorative perse dal familiare caregiver e gli esborsi che si sono resi necessari da parte della famiglia per assistenza privata.

\section{RISULTATI}

In Tabella 2 riportiamo il numero di interventi sanitari effettuati nei tre periodi di osservazione, il relativo costo unitario e il bilancio economico per mese di osservazione $[3,4]$.
Come presentato in Tabella 2, i costi per mese nei tre periodi di osservazione dimostrano che il Centro Diurno, a fronte di un indubbio vantaggio clinico testimoniato dalla variazione dei punteggi delle scale di valutazione MMSE (Mini Mental State Examination), ADL (Activity of Daily Leaving), IADL (Instrumental Activity of Daily Leaving) (Tabella 3), non determina un innalzamento dei costi rispetto all'assistenza territoriale.

\begin{tabular}{lccc}
\hline & T1 & T2 & T3 \\
\hline MMSE & 14,7 & $16,2^{*}$ & $12,7^{*}$ \\
ADL & 2,4 & $1,4^{*}$ & $2,8^{*}$ \\
IADL & 5,7 & $4,9^{*}$ & $6,7^{*}$ \\
\hline
\end{tabular}

$* p<0,05$ rispetto a $T 1$

T1: ingresso $C D$; $T 2$ : dimissione $C D$;

T3: fine dell'osservazione
Tabella 3

Variazione dei punteggi totali delle scale di valutazione clinica 


\section{Tabella 4}

Carichi e costi per la famiglia

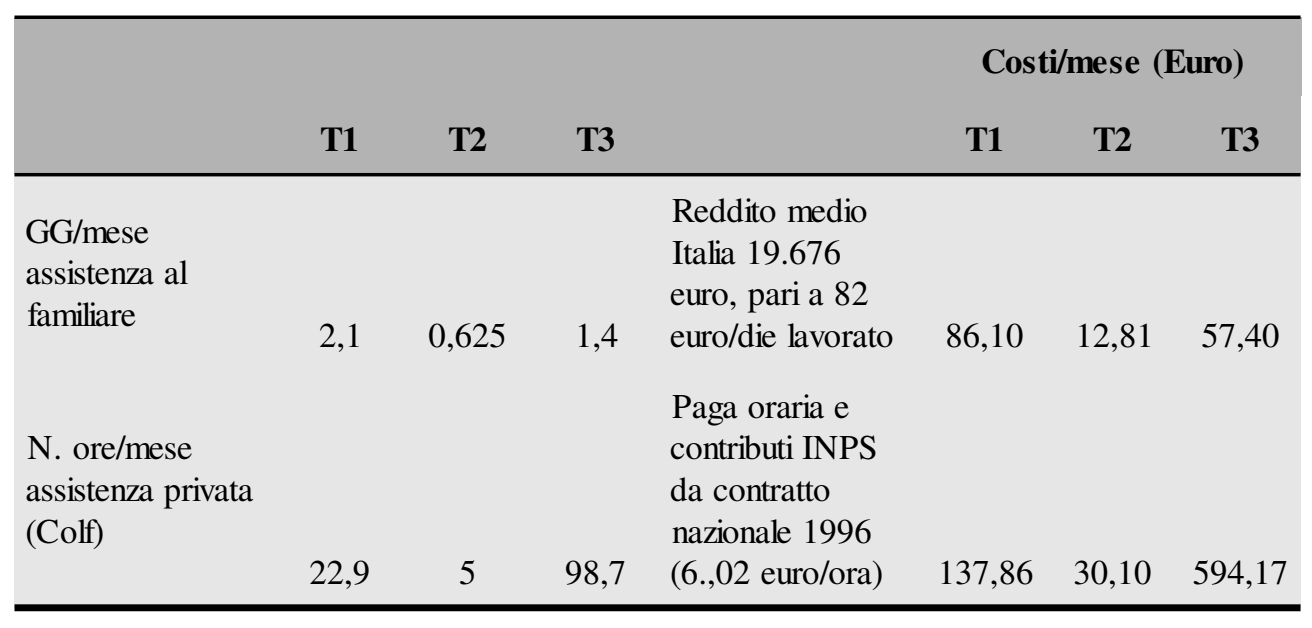

T1: ingresso CD; T2: dimissione CD; T3: fine dell'osservazione
Anzi, anche escludendo dal confronto il costo relativo ad un unico ricovero verificatosi nel periodo post Centro Diurno, si evidenzia che questa struttura è in grado di ridurre significativamente l'assistenza territoriale, portando ad una significativa riduzione dei costi sanitari globali.

In Tabella 4 riportiamo, infine, i costi relativi ai carichi familiari. Assegnando al familiare lavoratore un valore di reddito medio Italia e riconoscendo per l'assistenza privata una paga oraria come da Contratto Nazionale COLF (1996), si evidenzia che nel periodo di frequentazione del Centro Diurno i carichi familiari crollano vistosamente sia in termini di attività svolte per l'assistenza del paziente sia in termini economici.

\section{DISCUSSIONE}

La nostra analisi ha preso le mosse da una recente esperienza di studio osservazionale svolta presso la ASL 1 di Città di Castello. Le prestazioni considerate sono state erogate dalla suddetta ASL in un periodo di osservazione di 8 mesi.

Scopo della conversione economica delle variabili di consumo sanitario che abbiamo svolto è quello di verificare se il Centro Diurno possa essere considerato efficace strumento di razionalizzazione della spesa, oltre che efficace strumento di assistenza clinica dei pa- zienti affetti da Alzheimer probabile (come dimostrato dallo studio cui ci riferiamo) $[5,7]$.

Rispetto ai periodi di osservazione precedente e successivo a quello di frequentazione del Centro Diurno, l'accesso a questa struttura ha determinato un generale miglioramento delle condizioni cliniche dei pazienti (Tabella 3) [5], cui è corrisposta una significativa riduzione della richiesta di interventi sanitari, con conseguente abbattimento dei costi sanitari. Tale abbattimento è risultato tanto importante da bilanciare i costi di gestione del Centro Diurno stesso, grazie anche ad una minima contribuzione degli assistiti (retta di 10 euro/ die).

Nel periodo di accesso al Centro Diurno si è notata anche una riduzione importante dei carichi di assistenza da parte dei caregivers familiari. Evidente soprattutto l'abbattimento dei costi per forme di assistenza privata che, in questo caso, abbiamo attribuito essenzialmente a forme di supporto da parte di personale COLF specializzato nell' assistenza alla persona.

I risultati dello studio sono quindi senz'altro a favore dell'investimento da parte delle ASL nei Centri Diurni, soprattutto quando le infrastrutture possano essere messe a disposizione con forme di comodato/affitto gratuito come nel presente caso. Inoltre è da sottolineare il fatto che tali strutture assolvono anche ad una importantissima funzione sociale di sostegno alle famiglie, ancora oggi troppo spesso lasciate sole di fronte a questa malattia. 


\section{BIBLIOGRAFIA}

1. www.istat.it/Anotizie/Aaltrein/statinbrev/prevpop00/index.html

2. w3.uniroma1.it/fisiofarm/sifeit/testi/Bruno/sld010.htm

3. Fattore $\mathrm{G}$ et al. I costi delle prestazioni in psichiatria: un'analisi condotta in un CPS pubblico in Lombardia. Epidemiologia e psichiatria Sociale, 1997, vol. 6(2): 139-147.

4. Garattini L. et al. Analisi dei costi per divisione su un campione di 9 presidi ospedalieri. ASI, 1996, n. 10 : 33-37.

5. Studio osservazionale per la valutazione del carico assistenziale e familiare della demenza in pazienti con accesso ad un Centro Diurno. Psichiatria e Psicoterapia, 2004, supplemento in pubblicazione.

6. Trabucchi M. Le demenze. Utet Editore, Giugno 2002, pagg. 639-655.

7. Maj M, Sartorius N. Demenza. Seconda edizione, 2004, CIC Edizioni Internazionali. 